\title{
STUDY OF HARMONIC DETECTION METHODS UNDER NON-IDEAL CONDITIONS IN SHIP POWER NETWORK
}

\author{
Xiao-Yan Xu, Prof. ${ }^{1}$ \\ Janusz Mindykowski, Prof. ${ }^{2}$ \\ Tomasz Tarasiuk, Prof. ${ }^{2}$ \\ Chen Cheng, M.Sc. ${ }^{1}$ \\ 1) Shanghai Maritime University, Shanghai, China \\ 2) Gdynia Maritime University, Gdynia, Poland
}

\begin{abstract}
An improved harmonic detection method based on average arithmetic is proposed. According to the research results, the designed solution uses an LPF (low-pass-filter) and a mean value module connected in series instead of the conventional mean value module, and simultaneously, a three-phase voltage phase-locked module instead of commonly used PLL (phase lock loop) module is applied in order to reduce the influence caused by three-phase distorted voltage and rapid variation of load. The experimental results show that the application of this solution leads to increase in the accuracy of harmonics detection for distorted three-phase voltage and rapid variation of load.
\end{abstract}

Keywords: distorted three-phase voltage; rapid-variation load; harmonic detection; average arithmetic

\section{INTRODUCTION}

With the development of power electronic devices in recent decades, more and more power converters are applied onboard, and the ship electric propulsion system is the representative example. But the fact is, power quality in marine electric power networks is becoming worse and worse, especially the level of harmonics increases. For example, in the case of Queen Mary 2 [10], the bulky ship has suffered a failure of harmonic passive filter, which resulted in explosion and damage of the surrounding electrical panels, and finally a total black-out of the vessel. In this case, the only protection against catastrophic failure of the capacitors being a part of harmonic passive filter, was a current imbalance detection system, which after the accident was found to be inoperable [10]. Anyway, a problem how to detect and compensate harmonic components with appropriate accuracy in a real time is one of the most important questions concerning a problem of power quality in ship networks. This question is fundamental from the point of view active or hybrid power filters control [9], [15]. Conventional harmonic detecting method is based on the theories such as instantaneous reactive power theory, Fourier transform or adaptive algorithms. The

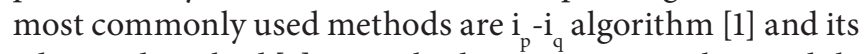
advanced method [8]. A method using a mean value module to replace LPF is described in [6] and [9] to improve the accuracy and reduce the response time of harmonic detection. The methods described in [1] and [9] using $i_{p}-i_{q}$ algorithm without PLL are proposed to solve the problem caused by asymmetrical load. In [16] and [17], symmetrical component method is proposed for positive sequence voltage and current measurement. But in the case of distorted three-phase voltage, these methods sometimes cannot detect positive sequence component accurately. And sometimes their accuracy may be influenced by the variation of the fundamental current. To reduce the influence of distorted three-phase voltage, a new three-phase PLL circuit is proposed in [7]. But using this circuit, the response time is longer. In [14], a PI controller is added to the detection system. In [12], a new PLL circuit is proposed for compensation of error which is caused by system delay. In this paper, a improved harmonic current detection method is discussed under the circumstances of distorted three-phase voltage - with unbalanced initial phase angles and amplitudes in the presence of rapid variation of load. In shorthand, an advanced PLL circuit is proposed for detection of the positive sequence voltage completed by a module consisting of a LPF and a mean module that are connected in series. It is to supersede the conventional LPF.

\section{HARMONIC DETECTION METHOD WITH MEAN VALUE MODULES}

\section{PRINCIPLE OF HARMONIC DETECTION METHOD WITH MEAN VALUE MODULES}

The harmonic detection method with mean value modules is graphically presented in Fig. 1. It applies $i_{p}-i_{q}$ algorithm but LPF module is replaced by mean value modules. The 
mean value obtained by simple integration is the DC value corresponding to the fundamental current. In an ideal power network, its detection time is only $1 / 6$ cycle [6]. Its principle analysis is as follows. Assuming that the three-phase load current is,

$$
\left\{\begin{array}{l}
i_{a}=\sum_{n=1}^{\infty} I_{n} \sin \left(n \omega t+\varphi_{n}\right) \\
i_{b}=\sum_{n=1}^{\infty} I_{n} \sin \left[n(\omega t-2 \pi / 3)+\varphi_{n}\right] \\
i_{c}=\sum_{n=1}^{\infty} I_{n} \sin \left[n(\omega t+2 \pi / 3)+\varphi_{n}\right]
\end{array}\right.
$$

where, $I_{n}$ and $\varphi_{n}$ are the amplitude and initial phase angle of the nth harmonic current. The sine and cosine signals corresponding to the voltage of phase $\mathrm{A}$, sin $\omega t$ and cos $\omega \mathrm{t}$ can be detect by the PLL. They form matrix $\mathrm{C}$ which is showed in Fig.1,

$$
C=\left[\begin{array}{cc}
\sin \omega t & -\cos \omega t \\
-\cos \omega t & -\sin \omega t
\end{array}\right]
$$

According to the scheme, the formulas of $\mathrm{i}_{\mathrm{p}}$ and $\mathrm{i}_{\mathrm{q}}$ are,

$$
\left\{\begin{array}{l}
i_{p}=\sqrt{\frac{2}{3}} \sum_{n=1}^{\infty} I_{n}\left[\sqrt{3} \sin \frac{2}{3} n \pi \cos \omega t \cos \left(n \omega t+\varphi_{n}\right)+\left(1-\cos \frac{2}{3} n \pi\right) \sin \omega t \sin \left(n \omega t+\varphi_{n}\right)\right] \\
i_{q}=\sqrt{\frac{2}{3}} \sum_{n=1}^{\infty} I_{n}\left[\sqrt{3} \sin \frac{2}{3} n \pi \sin \omega t \cos \left(n \omega t+\varphi_{n}\right)+\left(1-\cos \frac{2}{3} n \pi\right) \cos \omega t \sin \left(n \omega t+\varphi_{n}\right)\right]
\end{array}\right.
$$

Here, $i_{p}$ is taken for example; when $n=1$,

$$
i_{p}=\sqrt{\frac{3}{2}} I_{1} \cos \varphi_{1}=\overline{i_{p}}
$$

when $\mathrm{n}=3 \mathrm{k} \quad(\mathrm{k}=1,2,3 \ldots)$,

$$
i_{p}=0
$$

when $\mathrm{n}=3 \mathrm{k}+1 \quad(\mathrm{k}=1,2,3 \ldots)$,

$$
i_{p}=\sqrt{\frac{3}{2}} I_{n} \cos \left(3 k \omega t+\varphi_{n}\right)
$$

when $n=3 k-1 \quad(k=1,2,3 \ldots)$,

$$
i_{p}=-\sqrt{\frac{3}{2}} I_{n} \cos \left(3 k \omega t+\varphi_{n}\right)
$$

And $i_{q}$ can be obtained in the same way. In three-phase symmetry circuit, even order harmonics do not exist. The system only contains harmonic currents of 5 th, 7th, 11th and so on. So it can can be stated that only components $\mathrm{n}=6 \mathrm{k} \pm 1(\mathrm{k}=1,2,3 \ldots)$ would exist. Then ip and iq become,

$$
\left\{\begin{array}{l}
i_{p}=\overline{i_{p}} \pm \sqrt{\frac{3}{2}} \sum_{n=2}^{\infty} I_{n} \cos \left(6 k \omega t+\varphi_{n}\right) \\
i_{q}=\overline{i_{q}}-\sqrt{\frac{3}{2}} \sum_{n=2}^{\infty} I_{n} \sin \left(6 k \omega t+\varphi_{n}\right)
\end{array}\right.
$$

After integrating the above formulas for $1 / 6$ cycle, the fundamental component of $i_{p}$ and $i_{q}$ are obtained.

$$
\left\{\begin{array}{l}
\overline{i_{p}}=\frac{6}{T} \int_{t-\frac{T}{6}}^{t} i_{p} d t \\
\overline{i_{q}}=\frac{6}{T} \int_{t-\frac{T}{6}}^{t} i_{q} d t
\end{array}\right.
$$

After transforming them into three phase, fundamental currents iaf, ibf and icf are detected. And the harmonic component can be obtained.

\section{ERROR ANALYSIS}

As shown in Fig.1, the phase of sine \& cosine signal detected by method with mean value modules is determined by $u_{a}$. When three-phase voltage is asymmetrical and contains harmonic components, it has positive sequence component and negative sequence component. Detected by the PLL circuit, as shown in Fig.1, the phase of sine signal is the same as that of $\mathrm{u}_{\mathrm{a}}$, but differs by $\theta$ from its positive sequence component. The exact signal are $\sin (\omega t+\theta)$ and $\cos (\omega t+\theta)$. Calculating with the matrix $\mathrm{C}$ formed by them, the detection of fundamental active and reactive current will be influenced. Moreover, in the operation process of the APF (active power filter) from sampling of filtering signal to inverter output (Fig. 2), there exists sampling delay, transformer phase delay, signal processing delay and inverter delay of main circuit [3]. If the time delay is not compensated, the accuracy of detection will be very poor, and even the harmonic problem becomes more serious. That is, phase preprocessing during detection is necessary. But when load varies, the conventional detection method with mean modules may be readily disturbed by changing currents.

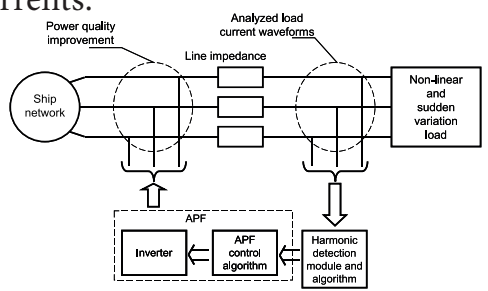

Fig. 2 Principle block diagram of the considered ship network, active power filter and its control

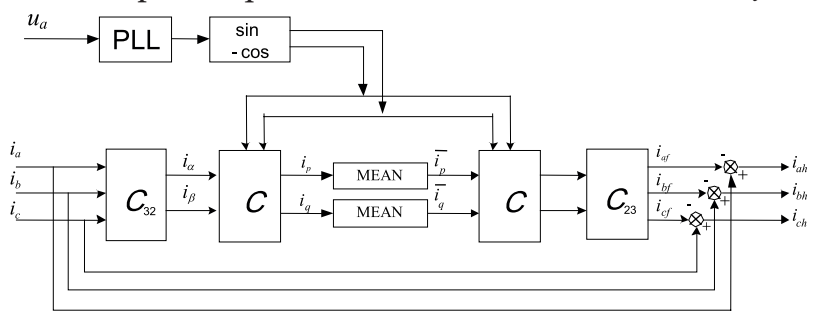

Fig.1 Block diagram of harmonic detection method based on $i_{p}-i_{q}$ concept with mean value modules 


\section{PRINCIPLE OF PROPOSED HARMONIC DETECTION METHOD}

According to the above error analysis, an improved harmonic detection method is proposed. Its scheme (block diagram) is shown in Fig. 3.

The proposed solution is able to detect positive sequence component of three-phase voltage when it is distorted and asymmetrical. Its principle analysis is as follows. Assume that the asymmetrical three-phase voltage is,

$$
\left\{\begin{array}{l}
u_{a}=\sum_{n=1}^{\infty} U_{n}^{+} \sin \left(n \omega t+\varphi_{u_{n}^{+}}\right)+\sum_{n=1}^{\infty} U_{n}^{-} \sin \left(n \omega t+\varphi_{u_{n}^{-}}\right) \\
u_{b}=\sum_{n=1}^{\infty} U_{n}^{+} \sin \left[n(\omega t-2 \pi / 3)+\varphi_{u_{n}^{+}}\right]+\sum_{n=1}^{\infty} U_{n}^{-} \sin \left[n(\omega t+2 \pi / 3)+\varphi_{u_{n}^{-}}\right](\mathbf{1 0}) \\
u_{c}=\sum_{n=1}^{\infty} U_{n}^{+} \sin \left[n(\omega t+2 \pi / 3)+\varphi_{u_{n}^{+}}\right]+\sum_{n=1}^{\infty} U_{n}^{-} \sin \left[n(\omega t-2 \pi / 3)+\varphi_{u_{n}^{-}}\right]
\end{array}\right.
$$

where, $\mathrm{U}$ represents voltage amplitude, $\mathrm{n}$ represents harmonic order, $\varphi$ represents initial angle, and (+,-) represent positive and negative sequence components.

When voltage signal passes BPF, the output signals are $\mathrm{u}_{\mathrm{af}}, \mathrm{u}_{\mathrm{bf}}$ and $\mathrm{u}_{\mathrm{cf}}$. Then the output signals are calculated by symmetrical component theory, all asymmetrical threephase vectors are divided into three parts - positive sequence component, negative sequence component and zero-phase sequence component. The formulas are,

$$
\left[\begin{array}{l}
f_{a} \\
f_{b} \\
f_{c}
\end{array}\right]=\left[\begin{array}{l}
f_{a}^{+}+f_{a}^{-}+f_{a}^{0} \\
f_{b}^{+}+f_{b}^{-}+f_{b}^{0} \\
f_{c}^{+}+f_{c}^{-}+f_{c}^{0}
\end{array}\right]=\left[\begin{array}{ccc}
1 & 1 & 1 \\
a^{2} & a & 1 \\
a & a^{2} & 1
\end{array}\right]\left[\begin{array}{l}
f_{a}^{+} \\
f_{a}^{-} \\
f_{a}^{0}
\end{array}\right]
$$

where, $a=e^{j 120^{\circ}}, a^{2}=e^{j 240^{\circ}}$, and $f_{\mathrm{a}}, f_{\mathrm{b}}, f_{\mathrm{c}}$ are unsymmetrical three-phase vectors [17]. Then the above formula is transformed into,

$$
\left[\begin{array}{l}
f_{a}^{+} \\
f_{a}^{-} \\
f_{a}^{0}
\end{array}\right]=\left[\begin{array}{ccc}
1 & 1 & 1 \\
a^{2} & a & 1 \\
a & a^{2} & 1
\end{array}\right]^{-1}\left[\begin{array}{l}
f_{a} \\
f_{b} \\
f_{c}
\end{array}\right]=\frac{1}{3}\left[\begin{array}{ccc}
1 & a^{2} & a \\
1 & a & a^{2} \\
1 & 1 & 1
\end{array}\right]\left[\begin{array}{l}
f_{a} \\
f_{b} \\
f_{c}
\end{array}\right]
$$

Similar method is applied to define the matrix T - in Fig.3 - as,

$$
T=\left[\begin{array}{ccc}
1 & a^{2} & a \\
1 & a & a^{2} \\
1 & 1 & 1
\end{array}\right]
$$

The positive sequence component of fundamental voltage can be readily detected. Its formula is,

$$
u_{a f}^{+}=\left(u_{a f}+a^{2} u_{b f}+a u_{c f}\right) / 3
$$

According to the above theory, the calculation of $\mathrm{u}_{\mathrm{bf}}$ need a $240^{\circ}$ phase shift, and $120^{\circ}$ for $\mathrm{u}_{\mathrm{cf}}$. The time delays at least $2 \pi / 3$. Using Formula (15),

$$
\left\{\begin{array}{l}
u_{b f} e^{j 240^{\circ}}=-u_{b f} e^{j 60^{\circ}} \\
u_{c f} e^{j 120^{\circ}}=-u_{c f}+u_{c f} e^{j 60^{\circ}}
\end{array}\right.
$$

Formula (14) becomes,

$$
u_{a f}{ }^{+}=\left(u_{a f}-u_{b f} e^{j 60^{\circ}}-u_{c f}+u_{c f} e^{j 60^{\circ}}\right) / 3
$$

So, signal processing method as shown in formula (16) is able to save time. After getting the positive sequence component of fundamental voltage, the sine signal $\sin \left(\omega \mathrm{t}+\varphi \mathrm{u}_{\mathrm{n}}^{+}\right)$and the cosine signal $\cos \left(\omega \mathrm{t}+\varphi \mathrm{u}_{\mathrm{n}}^{+}\right)$can be easily found. The matrix $\mathrm{C}$ as formed is,

$$
C=\left[\begin{array}{cc}
\sin \left(\omega t+\varphi_{u_{n}^{+}}\right) & -\cos \left(\omega t+\varphi_{u_{n}^{+}}\right) \\
-\cos \left(\omega t+\varphi_{u_{n}^{+}}\right) & -\sin \left(\omega t+\varphi_{u_{n}^{+}}\right)
\end{array}\right]
$$

Moreover, because of the time delay caused by the system itself, as shown in Fig. 2, compensation module as compensatory angle is added to make up for it. Assuming that the operation of APF is with a time delay equal to $\Delta t$, there is a delay angle equals to $\omega \Delta \mathrm{t}$. After the compensation module is applied, the new matrix $\mathrm{C}_{\Delta \mathrm{t}}$ is able to guarantee the identity of the phase after transformation. The matrix $\mathrm{C}_{\Delta t}$ is,

$$
C_{\Delta t}=\left[\begin{array}{cc}
\sin \left(\omega t+\varphi_{u_{n}^{+}}+\omega \Delta t\right) & -\cos \left(\omega t+\varphi_{u_{n}^{+}}+\omega \Delta t\right) \\
-\cos \left(\omega t+\varphi_{u_{n}^{+}}+\omega \Delta t\right) & -\sin \left(\omega t+\varphi_{u_{n}^{+}}+\omega \Delta t\right)
\end{array}\right]
$$

And the fundamental currents after compensation are,

$$
\left[\begin{array}{l}
i_{a f} \\
i_{b f} \\
i_{c f}
\end{array}\right]=C_{32}{ }^{-1} C_{\Delta t}\left[\begin{array}{l}
\overline{i_{p}} \\
\overline{i_{q}}
\end{array}\right]
$$

The system time delay angle is predicted by theoretical analysis of configuration shown in Fig 2. There is a difference between theoretical result and the result obtained in practical operation. So the fundamental current detected by this method is different from practical one. In practical operation, repeated test is necessary for finding the accurate time delay.

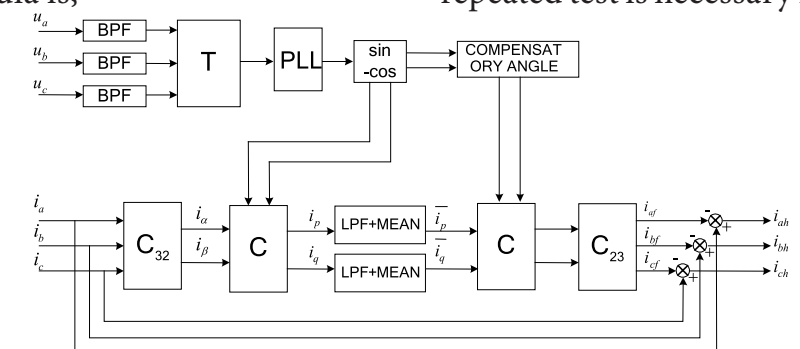

Fig.3 Block diagram of harmonic detection method with a three-phase voltage phase-locked module as well as low-pass filter and mean modules connected in series, $B P F$ - band-pass-filter 


\section{SIMULATION}

\section{SIMULATION MODEL OF THE SHIP NETWORK UNDER NON-IDEAL CONDITIONS}

Simulation was carried out wing Matlab / Simulink environment, applied for the configuration of ship electrical power system resulting from Fig.2. A comparative analysis of level of the waveform distortion and time of response of harmonic detection for the active power filtering was carried out by using Mathcad environment for waveform distortion analysis. The parameters of load are $\mathrm{R}=20 \Omega$ and $\mathrm{L}=2 \mathrm{mH}$. According to the analysis of [12], it is assumed that the time delay of the system is $140 \mu \mathrm{s}$. That would be $2.52^{\circ}$ if it is expressed in angle (for $\mathrm{f}=50 \mathrm{~Hz}$ ). The value of positive sequence voltage is $220 \mathrm{~V}$. The initial phases of A phase voltage, $\mathrm{B}$ phase voltage, and C phase voltage are $25^{\circ},-115^{\circ}$, and $120^{\circ}$ respectively. Then, harmonic voltage of 5th order, 7 th order, and 11th order are added to the three phases. In Fig. 4 the waveforms of the distorted three-phase voltage before APF compensation are shown.

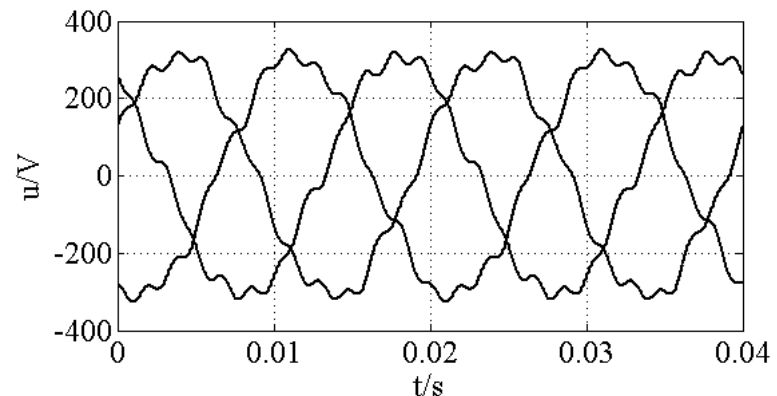

Fig.4 Distorted and asymmetrical three-phase voltage waveforms before APF compensation

\section{SIMULATION RESULTS UNDER DISTORTED THREE-PHASE VOLTAGE}

On the basis of the aforementioned model, comparative study by simulation experiments has been carried out, with usage of the three different detection methods, i.e. with mean modules, with mean modules and a three-phase voltage phaselocked module, and the proposed method. The experimental results are based on the assumption, that in all cases the same APF control method and load are used. An assessment of the distortion level of the analyzed waveform is based on the THD concept, in the meaning [4], [5]. Other distortion factors definitions are sometimes used [11]. Fig. 5 shows the results of harmonics detection by using the mean modules. Fig. 5(a) shows the positive sequence voltage and detected positive fundamental active current of phase A. Fig. 5(b) shows the detected fundamental current of phase A. It can be seen that a quite good waveform of the fundamental current can be detected after slightly above 0.01 s. Fig. 5 (c) shows the spectrum of detected fundamental current of phase A. Its THD value is $2.80 \%$. a)

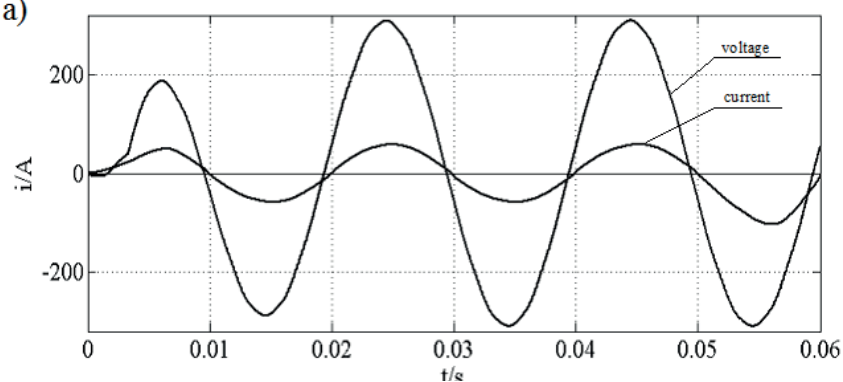

b)

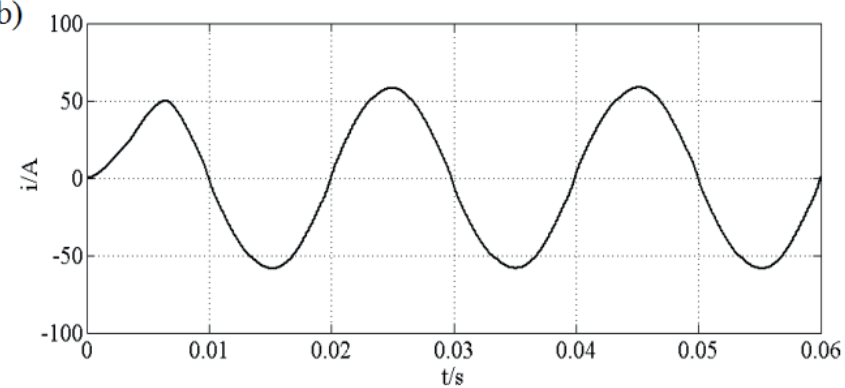

c)

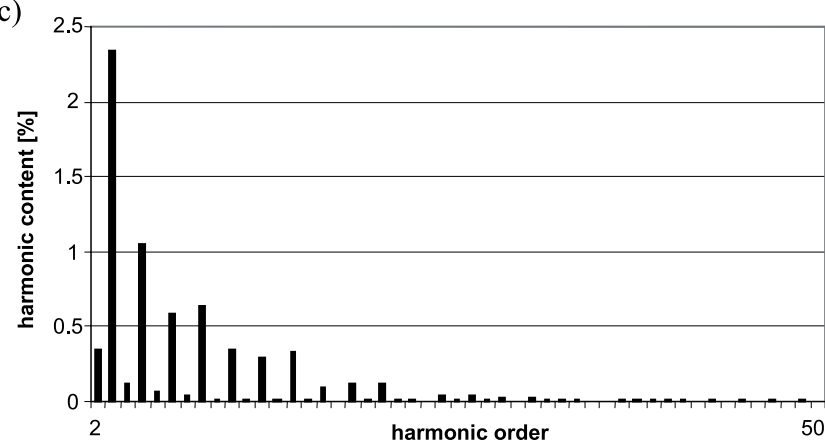

Fig.5 Simulation results of harmonic detection method with mean modules, (a) positive sequence voltage and detected positive fundamental active current of phase $A,(b)$ detected fundamental current of phase $A,(c)$ spectrum of detected fundamental current of phase $A$

Fig. 6 shows the results of harmonics detection by using the method with mean modules and a three-phase voltage phase-locked module. Fig. 6(a) shows the positive sequence voltage and detected positive fundamental active current of phase A. It is clear that there exists no phase difference. So the reactive compensation can be carried out accurately. Fig. 6(b) shows the detected fundamental current of phase A. It can be seen that a pretty ideal waveform of fundamental current can be detected after slightly above 0.01 s. Fig. 6 (c) shows the spectrum of detected fundamental current of phase A. Its THD value is $1.16 \%$.

Fig.7 shows the results of harmonics detection by using the proposed method. Fig.7 (a) shows the positive sequence voltage and detected positive fundamental active current of phase A. It's clearly that there exists no phase difference. So the reactive compensation can be carried out accurately. Fig.7 (b) shows the detected fundamental current of phase A. It can be seen that a pretty ideal waveform of fundamental current can be detected after one cycle. Fig.7 (c) shows the spectrum of detected fundamental current of phase A. Its THD value is $0.52 \%$. 
a)

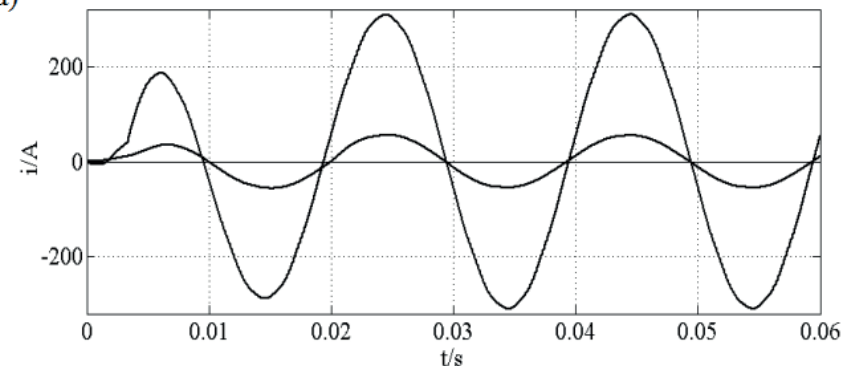

b)

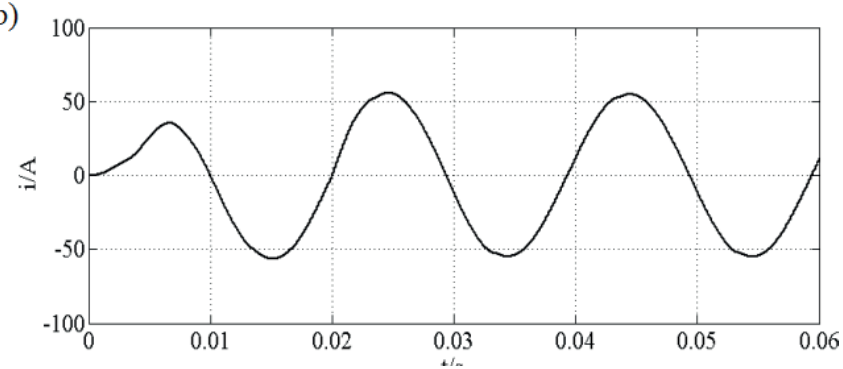

c)

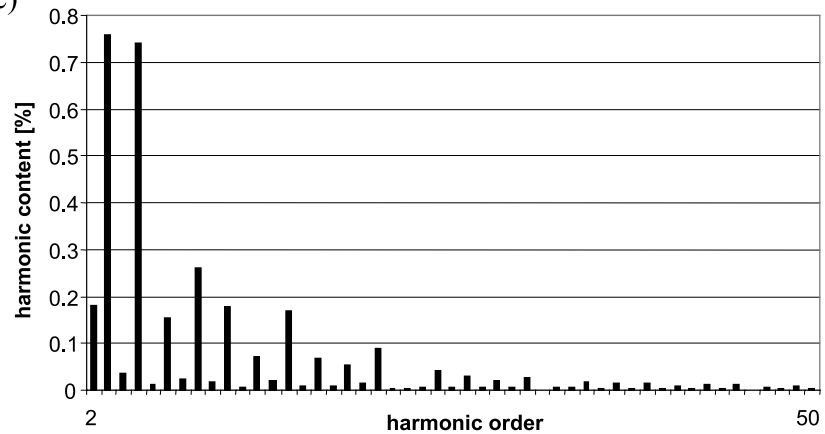

Fig.6 Simulation results of harmonic detection method with mean modules and a three-phase voltage phase-locked module, (a) positive sequence voltage and detected positive fundamental active current of phase $A,(b)$ detected fundamental current of phase $A$, (c) spectrum of detected fundamental current of phase $A$

Fig.7 shows the results of harmonics detection by using the proposed method. Fig.7 (a) shows the positive sequence voltage and detected positive fundamental active current of phase A. It's clearly that there exists no phase difference. So the reactive compensation can be carried out accurately. Fig.7 (b) shows the detected fundamental current of phase A. It can be seen that a pretty ideal waveform of fundamental current can be detected after one cycle. Fig.7 (c) shows the spectrum of detected fundamental current of phase A. Its THD value is $0.52 \%$.

After the comparison of the obtained results, it can be concluded that the accurate sine \& cosine signals can be detected by using the three-phase voltage phase-locked module. It helps with the accuracy of harmonic detection and reactive power compensation. The accuracy of the detection can be further improved by using a LP and a mean module connected in series. The THD value of the detected fundamental current dropped from $1.16 \%$ to $0.31 \%$. Though the THD value is noticeably lower, the response time increases more or less by $0.005 \mathrm{~s}$. a)

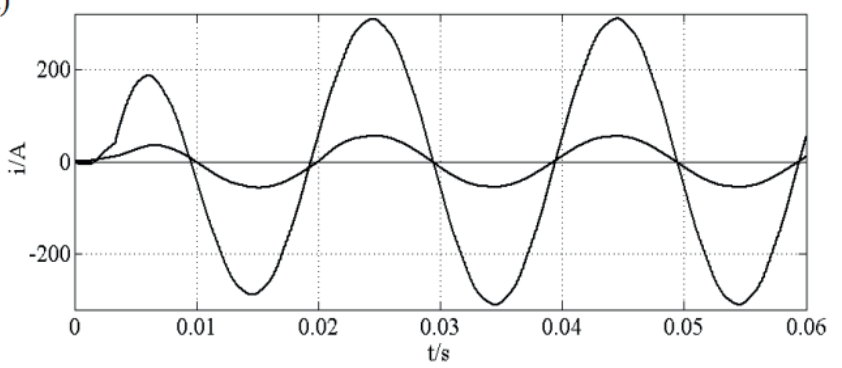

b)

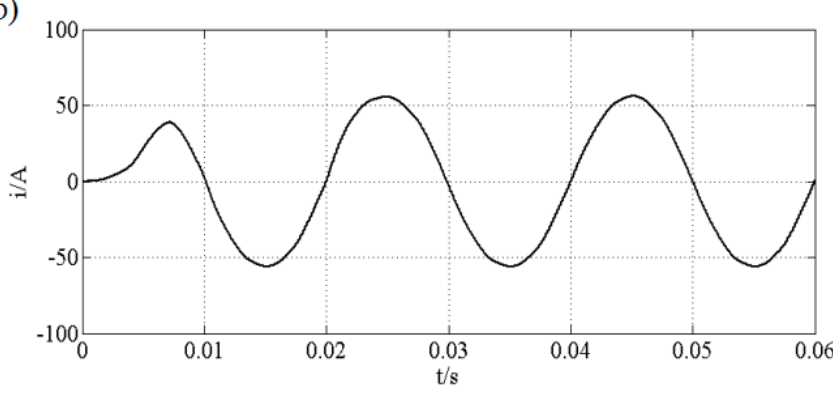

c)

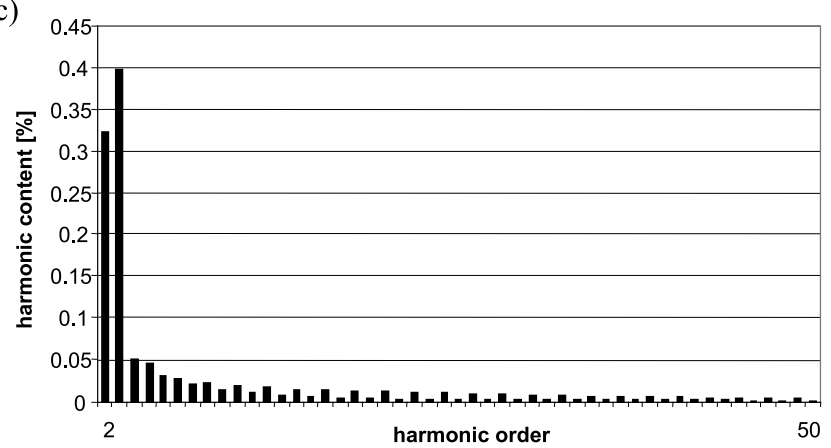

Fig.7 Simulation result of harmonic detection method with an LPF and a mean module connected in series and a three-phase voltage phase-locked module, (a) positive sequence voltage and detected positive fundamental active current of phase $A,(b)$ detected fundamental current of phase $A,(c)$ spectrum of detected fundamental current

\section{SIMULATION RESULTS UNDER DISTORTED THREE-PHASE VOLTAGE AND SUDDEN-VARIATION RAPID VARIATION OF LOAD}

An experiment of rapid variation of load is performed by load step change from $50 \%$ to $100 \%$ at 0.05 s time instant. That means the resistance drops from $20 \Omega$ to $10 \Omega$, and the inductance drops from $2 \mathrm{mH}$ to $1 \mathrm{mH}$. The resulting waveform of load current during the process is shown in Fig. 8.

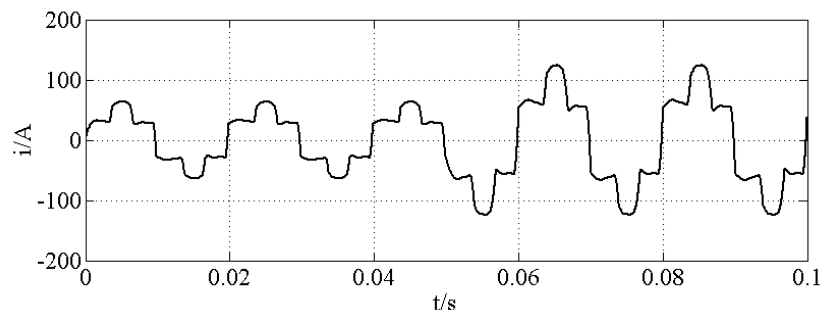

Fig. 8 Simulated current during load step change by $50 \%$ at 0.05 s time instant 
Next, the harmonic detection method with mean modules and the harmonic detection method with the proposed method are applied respectively. And the results are shown below. Fig. 9 shows the results obtained by using the harmonic detection method with mean modules. Fig. 9(a) shows the detected fundamental current of phase A. Fig. 9(b) and Fig. 9(c) are the spectrums of detected fundamental current of phase A before and half cycle after step load change. The THDs are $2.96 \%$ and $2.33 \%$ respectively. Further, the THD has been calculated for the cycle just after the step change ( $0.05 \mathrm{~s}$ to $0.07 \mathrm{~s}$ ). This has been equal to $8.37 \%$. Fig. 9 (d) shows the fluctuation of detected active component and reactive component respectively. It can been seen that fluctuation is very serious.

a)

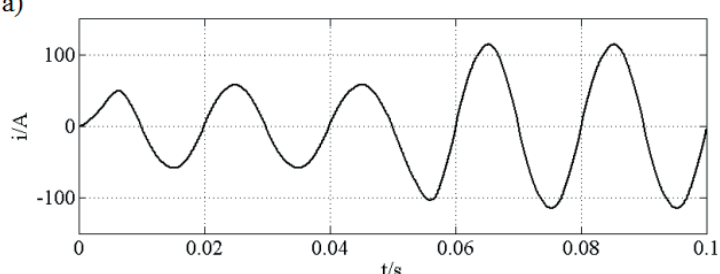

b)

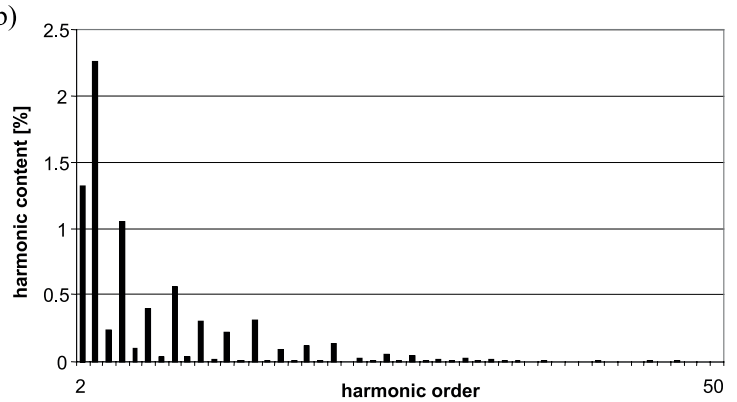

c)

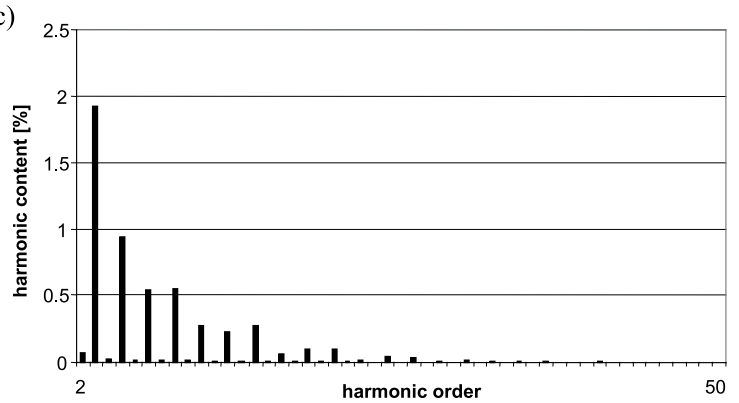

d)

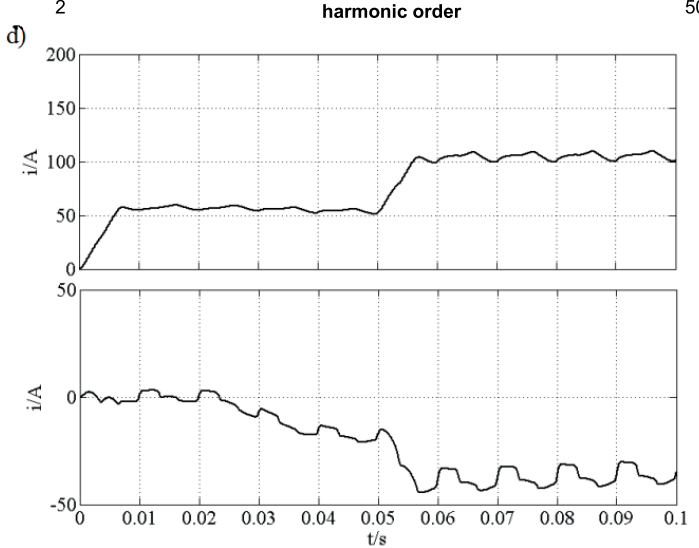

Fig.9 Simulation result of harmonic detection method with mean modules when load varies, (a) detected fundamental current of phase $A,(b)$ spectrum of detected fundamental current for one cycle before step load change $(0.03$ $s$ to $0.05 \mathrm{~s}),(\mathrm{c})$ spectrum of detected fundamental current for one cycle after step load change (0.06s to $0.08 \mathrm{~s})$, (d) detected active component and reactive component
Fig.10 shows the results obtained by using the proposed method. Fig.10(a) shows the detected fundamental current. Fig.10(b) and Fig.10(c) depict the spectra of detected fundamental current for one cycle before $(0.03 \mathrm{~s}$ to $0.05 \mathrm{~s})$ and one cycle after step load change $(0.07 \mathrm{~s}$ to $0.09 \mathrm{~s})$. The THDs of them are $1.31 \%$ and $0.5 \%$ respectively. Further, the THD has been calculated for the cycle just after the step change ( $0.05 \mathrm{~s}$ to $0.07 \mathrm{~s}$ ) and half cycle after load step change ( $0.06 \mathrm{~s}$ to $0.08 \mathrm{~s}$ ). These have been equal to $14.18 \%$ and $2.52 \%$. Fig. 10(e) shows the dynamic changing processing of detected active component and reactive component. The fluctuation is a little bit lower than in Fig. 9(d).

a)

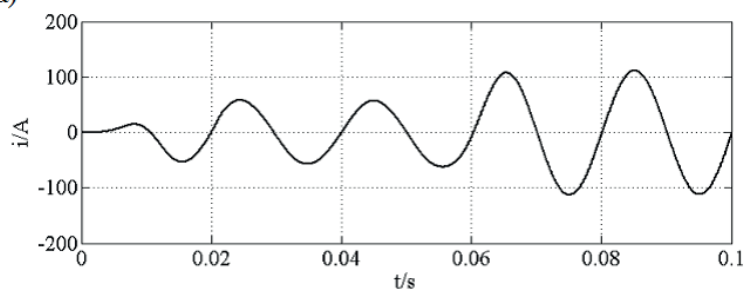

b)

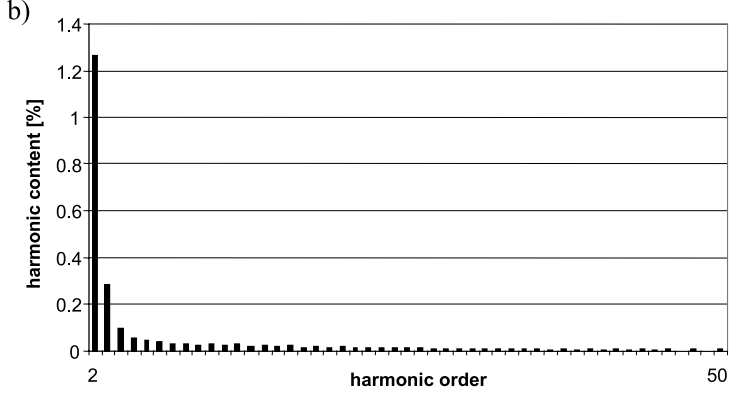

c)

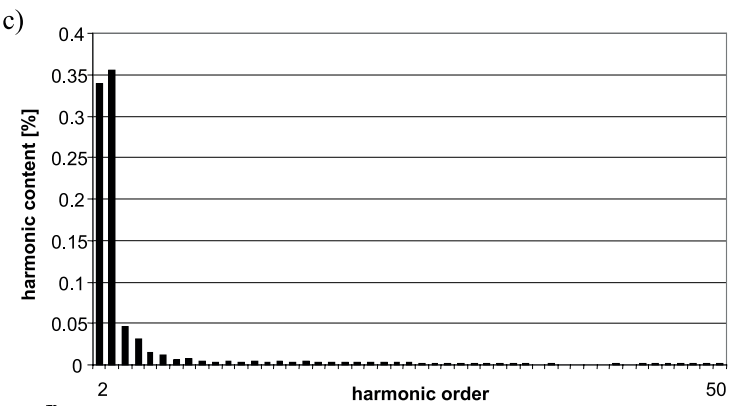

d)

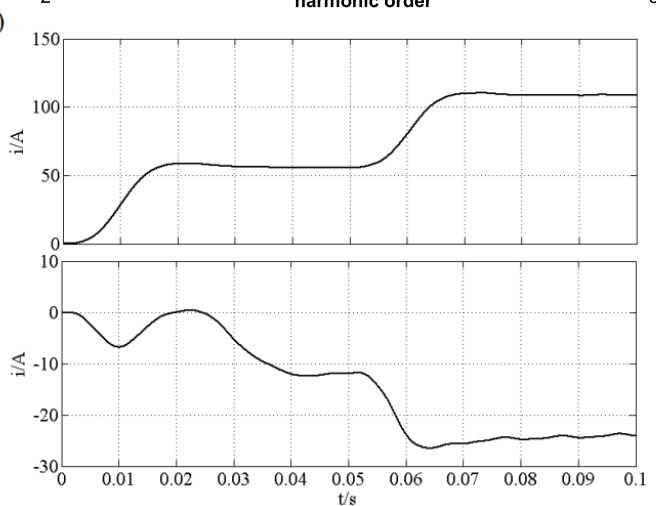

Fig.10 Simulation result of harmonic detection method with an LPF and a mean module connected in series and a three-phase voltage phase-locked module, when load varies, (a) detected fundamental current of phase $A,(b)$ spectrum of detected fundamental current for one cycle before step load change ( $0.03 \mathrm{~s}$ to $0.05 \mathrm{~s}),(\mathrm{c})$ spectrum of detected fundamental current for one cycle after step load change $(0.07 \mathrm{~s}$ to $0.09 \mathrm{~s})$, (d) detected active component and reactive component 
The careful comparison of the afore described experimental results leads to the following conclusions:

1. Within the first period immediately after the moment of load variation, THD value of fundamental current of phase A obtained by harmonic detection method with mean modules is $8.37 \%$, and that obtained by harmonic detection method with an LPF and a mean module connected in series and a three-phase voltage phase-locked module is significantly higher, i.e. $14.18 \%$.

2. It is also higher, if one considers its value half cycle after load step change, $2.33 \%$ versus $2.52 \%$. But after more than $0.01 \mathrm{~s}$, the THD value obtained by the harmonic detection method with an LPF and a mean module connected in series, and a three-phase voltage phase-locked module, started to drop quickly. After one cycle delay $(0.02 \mathrm{~s})$, it becomes $0.5 \%$. Whereas in the case of the harmonic detection method by only mean modules, THD reaches $2.33 \%$ and almost keep in this level. It's obvious that, though the response speed of the harmonic detection method an LPF and a mean module connected in series and a three-phase voltage phase-locked module, is not as quick as the response speed of the harmonic detection method with mean modules only, the former solution is more accurate. The difference of the two THD values of fundamental currents is $1.83 \%$.

3. The waveforms of active component and reactive component detected by the harmonic detection method with an LPF and a mean module connected in series reveals lower ripples of current components in comparison to the harmonic detection method with mean modules only. It shows that the proposed harmonic detection method has better performance.

\section{FINAL REMARKS}

The proposed solution is based on average arithmetic. It combines a LPF and a mean module connected in series with a three-phase voltage phase-locked module. It is a harmonic detection method with good performance under non-ideal conditions. According to the experimental results, four conclusions are given:

1. The conventional detection method based on average arithmetic cannot detect the positive sequence component and initial phase of the distorted voltage. The proposed solution solves this problem. And active power and reactive power compensation can be carried out accurately.

2. The THD value of fundamental current detected by conventional method is above $2.3 \%$. And the THD value of fundamental current detected by the proposed method is below $0.5 \%$. So that the proposed solution is more accurate.

3. The response time of conventional method is about 0.01 s. The response time of the proposed solution is below one cycle, but more or less 0.005 s slower.
4. When load suddenly changes, the detected by the proposed solution active and reactive current components have less ripples, which shows that the proposed harmonic detection method has better performance.

In shorthand, the proposed solution is able to overcome the shortcoming of the conventional harmonic detection method with mean modules when the two solutions are applied to detect current harmonics under the condition that 3-phase voltage is distorted or load varies suddenly. Simply put, the proposed solution leads to broadly understood higher detection accuracy. The downside of the proposed solution is that its response time is $0.005 \mathrm{~s}$ longer than that of conventional harmonic detection method based on average arithmetic. Further study will be undertaken to solve this problem.

\section{ACKNOWLEDGEMENT}

International S\&T Cooperation Program of China (2012DFG71850) \& Poland (No 35-20, 2013-2014 joint research project)

\section{BIBLIOGRAPHY}

1. Akagi H., Kanazawa Y., Nabae A., Instantaneous reactive power compensators comprising switching devices without energy storage components, IEEE Transactions on Industry Application 20 (3) (1984) 625-630

2. Ding Ju-Xia, Zhang Xiu-Feng. A harmonics-detection method without PLL and based on average theory in single-phase circuit [J]. Power System Protection and Control, 2010, 38(13): 26-30

3. Dong Tian-Bing, Cheng Han-Xiang, He Man-run. Time delay analyzing and the setting about the frequency compensation of active power Filter [J]. Electric Drive, 2011, 41(2): 24-27

4. EN Standard 50160. Voltage characteristics of electricity supplied by public distribution systems

5. IEC 61000-4-7:2007 General Guide on Harmonics and Interharmonics Measurements for Power Supply Systems and Equipment Connected Thereto

6. Jiang You-Hua, Cao Yi-long, Tang Zhong. Design and application of active power filter based on average arithmetic [J]. Power Electronics, 2010, 44(2): 42-43

7. Lu Zhen, Liang Guo-Lu, Lu Yu. Improved ip-iq harmonic detection method and simulation [J]. Power Electronics, 2012, 46(5): 81-83

8. Meng Jun-Xia, Zhou Zi-Guan, Li Guang-Hui. Detection of Distorted Current Based on ip-iq Method and Its Physical Realization [J]. Power System Technology, 2012, 36(5): 125-131 
9. Mindykowski J., Xu X., Tarasiuk T., A new concept of harmonic current detection for shunt active power filters control, Measurement 46 (2013) 4334-4341

10. Report of the investigation of the catastrophic failure of a capacitor in the aft harmonic filter room on board RMS Queen Mary 2 while approaching Barcelona on 23 September 2010, Marine Accident Investigation Branch, December, 2011

11. Tarasiuk T., Mindykowski J., An extended interpretation of THD concept in the wake of ship electric power system research, Measurement 45 (2012), 207-212

12. Tu Yong-Chang, Liu Jian-Gong, Wang Wei. Research on improved algorithm for harmonic current detection based on instantaneous reactive power theory [J]. Modern Electronics Technique, 2013, 36(3): 145-147

13. Wang Yi-Fei, Li Yun-Wei. Three-Phase Cascaded Delayed Signal Cancellation PLL for Fast Selective Harmonic Detection [J]. IEEE Transactions on industrial electronics, 2013, 60(4): 1452-1463

14. Xie Dong, Hui Jing. Application and research on modified ip-iq harmonic detection method [J]. Power Electronics, 2013, 47(1): 95-97

15. Xu X, Mindykowski J., Chen P., Study on Hybrid Filtering Solution for Marine Electric Network, Polish Maritime Research 2 (65) 2010. vol.17, pp.72-78

16. Yang Sheng-Yue, Wu Min, Huang Shen-Xi. A harmonic current detection method considering frequency shift [J]. Automation of Electric Power Systems, 2012, 36(7): 57-61

17. Yuan Xu-Feng, Cheng Shi-Jie, Wen Jin-Wu. An improved method of instantaneous symmetrical components and its detection for positive and negative sequence current [J]. Proceedings of the CSEE, 2008, 28(1): 52-58

18. Zhou Qing, Mao Ya-Hui, Zhao Yong-Bin. Improved harmonic detection method without PLL and LPF [J]. Electrical Measurement \& Instrumentation, 2012, 49(554): 27-31

\section{CONTACT WITH AUTHOR}

\author{
Xiao-Yan Xu \\ e-mail:xuxy@shmtu.edu.cn \\ Logistics Engineering Faculty, \\ Shanghai Maritime University \\ Shanghai 201306 \\ China
}

Janusz Mindykowski

e-mail: janmind@am.gdynia.pl

Marine Electrical Engineering Faculty Gdynia Maritime University 81-225 Gdynia

Poland

Tomasz Tarasiuk

e-mail: totar@am.gdynia.pl

Marine Electrical Engineering Faculty

Gdynia Maritime University

81-225 Gdynia

Poland

Chen Cheng

e-mail: losenessc@live.cn

Logistics Engineering Faculty

Shanghai Maritime University

Shanghai 201306

China 